\title{
Design and test of centrifugal disc type sowing device for unmanned helicopter
}

\author{
Cancan Song ${ }^{1,2}$, Zhiyan Zhou ${ }^{1,2,3^{*}}$, Xiwen Luo ${ }^{1,2}$, Yubin Lan ${ }^{1,2}$, Xingang $\mathrm{He}^{1,2}$, \\ Rui Ming ${ }^{1,2}$, Keliang Li ${ }^{1,2}$, Shahbaz Gul Hassan ${ }^{1,2}$ \\ (1. College of Engineering, South China Agricultural University / Guangdong Engineering Research Center for Agricultural Aviation \\ Application (ERCAAA), Guangzhou 510642, China; 2. National Joint Research Center for Precision Agricultural Aviation Application \\ Technology, Guangzhou 510642, China; 3. Key Laboratory of Key Technology on Agricultural Machine and Equipment \\ (South China Agricultural University), Ministry of Education, Guangzhou 510642, China)
}

\begin{abstract}
In China, it is difficult for manned aircraft to sow seeds in small and scattered plots, especially in areas including hills, swamps, telegraph poles, windbreaks, and residential areas; in such terrain, the sowing machinery cannot function properly. However, unmanned helicopters (UHs) are flexible enough to control and adapt to the complex environments that are not easily accessible by terrain sowing machinery and large agricultural aircraft, which have been widely used in agriculture. In this study, a centrifugal disc-type sowing device for an unmanned helicopter (CDTSDUH) was designed. The factors influencing the seed velocity when the seeds move away from the disc were explored by analyzing the forces of the seed acting on the sowing disc and the wind field of the UHs. The influential factors include the distance from the falling mouth to the center of the disc, the offset angle of the falling mouth, and the rotation speed of the disc. An orthogonal test was designed with the sowing width and the curvature of the sowing area as the indexes. The test results showed that the three factors mentioned above had a greater impact on the sowing width than the curvature of the seeding area. Moreover, the superior parameters of the disc were determined. It was also suggested that the above factor levels had little effect on the offset width of sowing. The results of the test conducted for studying the sowing uniformity of the CDTSDUH indicated that the maximum and minimum difference values among the number of particles of the sampling points in the forward direction was 11 and 8 , and the coefficient of variation of the number of particles in each row was more than $20 \%$, indicating less uniformity was achieved when sowing in the lateral (perpendicular to the forward direction) direction, as compared to that in the forward direction. This study determined the ideal values for the radius of particles position, the offsetting angle, and the disc speed of the sowing machinery designed. Furthermore, considering that there are so many factors that influence the manner in which a UH functions, more analysis results and test data of influencing factors need to be acquired by experiments. The uniformity of sowing needs to be further improved. The results provided some theoretical and experimental references for the technology research on the centrifugal disc-type sowing device for UHs.
\end{abstract}

Keywords: sowing, unmanned helicopter, aircraft sowing, centrifugal disc, uniformity, sowing disc DOI: $10.25165 /$ j.ijabe.20181102.3757

Citation: Song C C, Zhou Z Y, Luo X W, Lan Y B, He X G, Ming R, et al. Design and test of centrifugal disc type sowing device for unmanned helicopter. Int J Agric \& Biol Eng, 2018; 11(2): 55-61.

\section{Introduction}

The introduction of agricultural aviation technology opened up a new direction for the mechanization of crop planting ${ }^{[1,2]}$. The use of agricultural aircraft started worldwide many years ago, as a

Received date: $2017-08-25 \quad$ Accepted date: 2018-01-03

Biographies: Cancan Song, Master student, research interests: precision agriculture and agricultural aviation, Email: 737448145@qq.com; Xiwen Luo, Professor, research interests: agricultural equipment, precision agriculture and agricultural aviation, Email: xwluo@scau.edu.cn; Yubin Lan, PhD, Professor, research interests: precision agriculture and agricultural aviation, Email: ylan@scau.edu.cn; Xingang He, Master student, research interests: agricultural aviation, Email: 1096260969@qq.com; Rui Ming, Master student, research interests: agricultural aviation, Email: 502100347@qq.com; Keliang Li, Master student, research interests: remote sensing monitor of UAV. Email: 1218088661@qq.com; Shahbaz Gul Hassan, PhD, research interests: sensor design and image analysis, Email: Mhasan387@cau.edu.cn.

*Corresponding author: Zhiyan Zhou, Professor, research interests: agricultural aviation. College of Engineering, South China Agricultural University, Guangzhou 510642, China. Tel: +86-13560026139, Email: zyzhou@scau.edu.cn. large number of light aircraft were converted into agricultural aircraft for performing spraying operations ${ }^{[3]}$ after World War II. Then, many countries began to use a wide range of aircraft for planting and fertilization. Presently, at least $37 \%$ of rice fields in the United States are planted by airplanes, and $100 \%$ of rice topdressing works are performed by aircraft. According to statistics, there are more than 4000 agricultural aircraft currently used in America, which includes single rotors, twin rotors, and helicopters. Moreover, manned fixed-wing aircraft accounted for approximately $88 \%$ of agricultural aircraft ${ }^{[4]}$. In Japan, broadcast sowing was performed mainly using artificial and remote-controlled helicopters ${ }^{[5]}$. Other countries also employed artificial aircraft, mainly for large contiguous operation areas.

The main rice producing district in China, i.e., southern China, has many issues involving scatter, paddy fields, great height difference, depth of mud, excess rain. In particular, there is deep latent layer and poor drainage in cold leaching fields, one of the main low-yield paddy field areas. Cold leaching fields account for $44.2 \%$ of low-yield paddy field areas and $15.07 \%$ of the total paddy field areas in China. Planting in such terrain is difficult 
using sowing machinery ${ }^{[6,7]}$. In the rice stubble area of the Yangtze River Basin, compaction damage on the surface soil caused by large-scale ground machinery has an adverse effect on the emergence rate of the next season's crop. As a result, it is difficult for large machinery to start field operations. It is also difficult for small machinery to achieve a good sowing effect under the condition of straw incorporation ${ }^{[8]}$. Depending on the production conditions in the hilly drylands of the southwestern areas, small sowing machinery is used for sowing, but it is time-consuming and laborious ${ }^{[9]}$. In addition, there are 2.2 million hectares of coastal beach area ${ }^{[10]}$ and approximately 11 million hectares of swamp area in China ${ }^{[11]}$ continuously or dispersedly, where ground machinery is not suitable for sowing.

In some special areas, the general terrain sowing machinery cannot meet the requirements of production ${ }^{[12-14]}$. Recently, in the barren hills, large deserts contiguous to the area of Shaanxi, northeast and other places in China, aerial and other sowing operations have been carried out, with good economic and ecological benefits ${ }^{[15-17]}$. Although they offer high efficiency, unmanned agricultural aircraft (UAA) require special airports to take off and land. There is a need for UAA to work in limited and contiguous areas, but they cannot adapt to the complex and small dispersed areas, especially in some windbreaks, residential land, and areas with poles. In recent years, the flight control technology of unmanned helicopters (UHs) has developed rapidly owing to improvements in path planning operations, increase in safer landings, and quicker real-time responses to the operating environment ${ }^{[18]}$. All of these technologies provide the UHs with strong support in sowing operations. UHs are flexible to control and can fly at ultra-low altitudes without terrain constraints ${ }^{[19]}$. In addition, the UHs can be easily employed in places where terrain machinery and manned aircraft are not suitable.

Existing terrain disc-type sowing devices are mostly of the traction or hanging type, relying on ground machinery to provide power ${ }^{[20]}$. The UAA sowing devices rely mainly on the driver to manipulate the handle and control the seed falling mouth at the bottom of the aircraft. Terrain sowing devices and UAA sowing devices cannot be used directly on the UH because of the type, the small size of the $\mathrm{UH}$, and its ability to carry only limited load ${ }^{[21]}$. In order to study the sowing devices suitable for the $\mathrm{UH}$, as a supplement to the terrain sowing devices and UAA sowing devices, a centrifugal disc-type sowing device for an unmanned helicopter (CDTSDUH) was studied. The working principles, seed movement analysis, sowing width, and distribution were analyzed, and the parameters of sowing width and uniformity were optimized.

\section{Structure and working principle}

The CDTSDUH was mainly composed of an external force feed $^{[22]}$ and a light centrifugal disc. The traditional external force feed was optimized by increasing the groove width and diameter, which improved the speed, reduced the pulsation phenomenon ${ }^{[23]}$, and formed continuous particle flow. With an overall compact structure, lightweight metal materials, and large-torque light motor, this sowing device was more conducive when being used with the $\mathrm{UH}$ in low-altitude and high-speed operations. As shown in Figure 1, the seed particles fall into the centrifugal disc from the falling mouth through the external force feed and move to the edge of the disc via centrifugal force and friction; as a result, the seed particles get scattered, forming falling areas. The quantity and width of sowing can be adjusted according to the sowing height and speed of the UH.

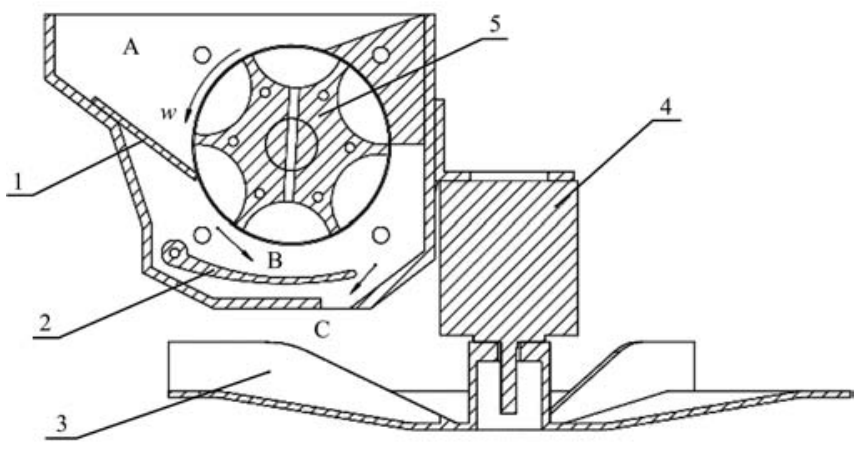

$\begin{array}{llll}\text { 1. Hairbrush } & \text { 2. Seed cup gate } \quad 3 \text {. Centrifugal disc } \quad \text { 4. Dynamo } \quad \text { 5. Seed roller }\end{array}$ $\begin{array}{lll}\text { A. filling area } & \text { B. seed flow layer } \quad \text { C. seed outlet }\end{array}$

Figure 1 Schematic diagram of centrifugal disc-type sowing device

\section{Design and analysis}

\subsection{Kinematic analysis of particles on centrifugal disc}

Grain particles were irregular and varied before touching the blades. The movements of the particles were complex, the trajectories were helix, and once they come into contact with the blades, they move along the blades, and finally get scattered away from the edge of the centrifugal disc, as shown in Figure 2. According to the theory of physics, it was found that a particle M falling on any point $\mathrm{A}$ of the centrifugal disc at zero initial velocity can achieve the velocity at the edge of the disk as depicted in the following formula (when neglecting the interaction between the particles) $)^{[24]}$ :

$$
V=\frac{\omega r}{\sin \beta}
$$

where, $V$ is the velocity of the particles leaving the disk, $\mathrm{m} / \mathrm{s} ; \omega$ is the angular velocity of the disk, $\mathrm{rad} / \mathrm{s} ; r$ is the distance between the particle on the centrifugal disc and the center of the disc, $\mathrm{m} ; \beta$ is the angle between the particle velocity and the particle in the diametrical direction, $\left(^{\circ}\right) ; \Psi$ is the angle between the position of the particles on the edge of the centrifugal disc and the forward direction, $\left({ }^{\circ}\right)$.

Therefore, the main influencing factors of the velocity of the particles from the centrifugal disc are $\omega, r$, and $\beta$.

\subsection{Kinematic analysis of particles in wind field}

After leaving the centrifugal disc, the particles mainly moved in the air flow, as they were affected by the wind field. Studying the distribution of the wind field can help in analyzing the movement of the particles in the wind field as well as the particle trajectory. The results showed that the distributions of the UH wind field were different in $X$-, $Y$-, and $Z$-directions (the $X$-direction represented the direction of in which the helicopter moves forward, the $Y$-direction represented the horizontal direction perpendicular to the forward direction of the $\mathrm{UH}$, and the $Z$-direction represents the vertical direction), the width of the wind field in the $X$-direction was significantly larger than that the $Y$-direction, and the $Z$-direction wind speed was the smallest ${ }^{[25]}$. As shown in Figure 2, the three-dimensional wind system was established, with the $X$-axis parallel to the forward direction of the $\mathrm{UH}$, the $Y$-axis perpendicular to the flight direction, and the $Z$-axis perpendicular to the ground.

$$
\begin{aligned}
X \text {-axis: } & F_{x}=m \frac{d V_{x}}{d t}, F_{x}=m k v_{x}^{2} \\
v_{x} & =w_{x}-V_{x}, V_{x}=V \cos
\end{aligned}
$$




$$
\begin{array}{cc}
Y \text {-axis: } & F_{y}=m \frac{d V_{y}}{d t}, F_{y}=m k v_{y}^{2} \\
& v_{y}=w_{y}-V_{y}, V_{y}=V \sin \theta \\
Z \text {-axis: } & F_{z}+m g=m \frac{d V_{z}}{d t}, F_{z}=m k v_{z}^{2} \\
& v_{z}=w_{z}-V_{z}
\end{array}
$$

where, $F(x, y, z)$ represents the forces of the airflow on the particles in three directions, $\mathrm{N} ; m$ is the particle mass, $\mathrm{g} ; V(x, y, z)$ represents the absolute velocities of the particles in the three directions $(\mathrm{m} / \mathrm{s})$, the initial values are $V_{x}=V \cos \theta, V_{y}=V \sin \theta$, and $V_{z}=0 ; K$ is the coefficient, relating to the relative velocity of the grain particles, the air flow, and grain characteristics; $W_{x, y, z}$ represents the velocities of the air flow in three directions; $V_{x, y, z}$ represents the relative velocities of the airflow and grain particles in the three directions; $\theta$ is the angle between the velocity of the grain at the edge of the disc and the $X$-axis, $\left({ }^{\circ}\right)$.

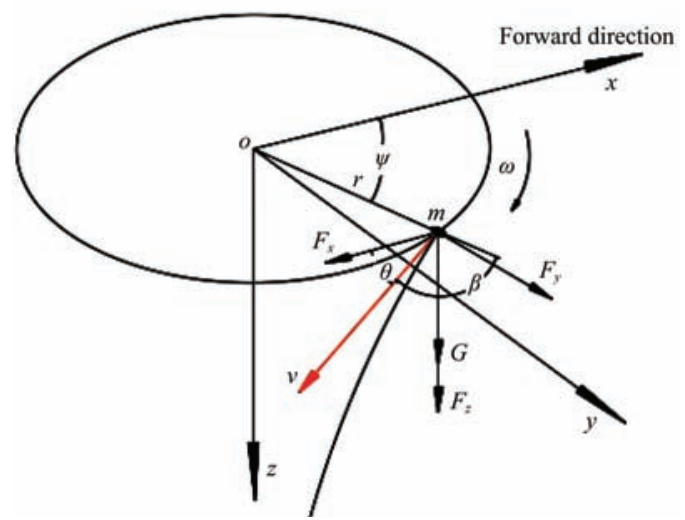

Figure 2 Diagram of particle motion off the centrifugal disc

During the falling process, the forces in the $X$ - and $Y$-directions affect the sowing width of the grain particles together, which benefits sowing. In particular, the sowing width of the grain particles increase more obviously when the wind speed is increased along the direction of the $Y$-axis. The force acting along the $Z$-axis on the grain particles affects landing time. When the velocity of the air flow is less than approximately $3 \mathrm{~m} / \mathrm{s}$, the air resistance is small when compared to the grain weight itself $^{[26]}$; however, the wind field under the UH fuselage has a considerable impact on the grain particle trajectory. For example, just about $0.5 \mathrm{~m}$ under the $\mathrm{UH}$ fuselage, the airflow velocity was more than $3 \mathrm{~m} / \mathrm{s}^{[27]}$. Therefore, the movement states and trajectories of the grain particles in the airflow were related to the velocity of the air flow in the rotor wind field, the velocity of the grain particles, the direction of the airflow, the initial velocity, and the direction angle of the grain particles entering the air flow field ${ }^{[28]}$.

\subsection{Sowing device integrated control for the UH}

\subsubsection{Sowing control system components}

As shown in Figure 3, the control part of the sowing device was mainly composed of the flight control system, the sowing decision system, the seeding motor and controller, the disk motor, the controller, and the human-computer interaction system. The flight control system was used to control the UH flight operations and for path planning ${ }^{[29]}$. The sowing decision system was used to control the extent of sowing. The sowing motor and the controller were used to control the grain particle sowing, response, and feedback sowing decision system, and the disk motor and the controller were used to control the sowing disc, control the sowing width, response, and feedback sowing decision system. The man-machine interaction system provided the control panel, which sets the parameters of the sowing device.

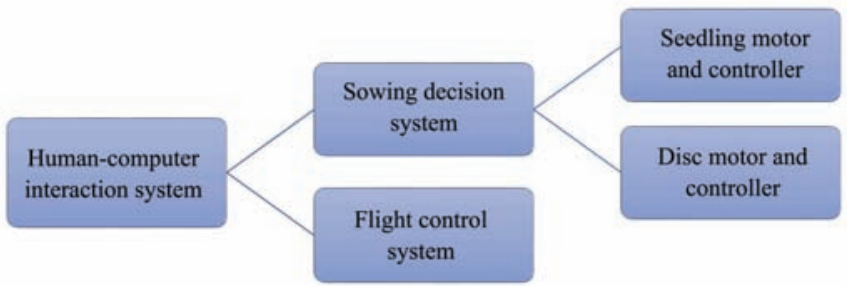

Figure 3 Diagram of the components of the sowing control system

\subsubsection{Working principle of the sowing control system}

Different rice varieties corresponded to different quantities when sowing. When sowing with the UH, path planning should be carried out and then, the UH operating altitude, motor speed, and speed of the disc motor need to be set. It was necessary to know the requirements of the sowing quantity and cultivated area to achieve the required sowing width in terms of the spread and uniformity. First, the UH is lifted to a predetermined height to start the disc motor and adjust it to the preset speed. Following this, the sowing motor is started and adjusted to the preset speed, by doing which the UH is controlled to sow.

\section{Sowing device parameters}

The sowing width was perpendicular to the direction of the UH An appropriate width is one of the important indexes in the design of the sowing device to increase the UH single flight coverage area and improve operational efficiency, considering that the UH has limited load carrying capacity and can fly for only a short duration. It was essential to study the effect of the factors of the sowing width to obtain the appropriate width. First, the sowing disc was tested on an indoor table with a height of $1 \mathrm{~m}$.

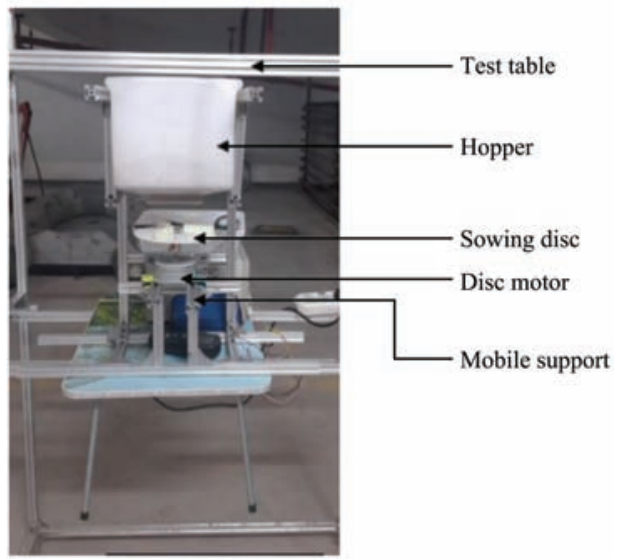

Figure 4 Test bed structure

As can be seen in Equation (1), the velocity of the grain particles away from the device was related to the rotational speed of the disc. The distance of the grain particles on the disc to the edge of the disc, the angle between the grain velocity, and the diametrical direction were related to the width. It was necessary to determine the optimal position of the mouth to find the appropriate spreading span. The three levels and three factors that were tested are listed in Table 1. The distribution of the locations of the falling mouth is shown in Figure 5. Because the radius of the falling point, the offset angle of the falling point, and the rotation speed of the disk shared no relationship, the interactions between the factors were neglected. 
Table 1 Three factors and three levels of the test

\begin{tabular}{ccccc}
\hline \multirow{2}{*}{ Levels } & Factor A & Factor B & \multirow{2}{*}{ Factor C } \\
\cline { 2 - 3 } & Radiuses of particles position $/ \mathrm{m}$ & & Offset angles $/\left(^{\circ}\right)$ & Speeds $/ \mathrm{r} \cdot \mathrm{min}^{-1}$ \\
\hline 1 & 18 & 0 & 500 \\
2 & 37 & 30 & 750 \\
3 & 55 & 60 & 1000 \\
\hline
\end{tabular}

Note: 1. the radius of the falling point refers to the grain particles falling on the disc to the center of the disc to the center of the distance; 2 . offset angle is the direction of the forward direction of the UH, grain placement along the radius of the direction.

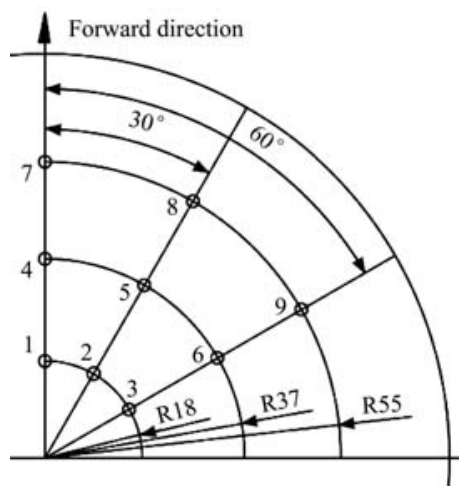

Figure 5 Distribution of seed falling positions

In order to ensure that test results are accurate, the impact of non-test factors can be excluded by ensuring the disc motor can be moved in the horizontal plane of two mutually perpendicular directions. The falling mouth of the hopper was rectangularly crossed, and the length from the mouth to the top of the disc was approximately 2-3 $\mathrm{mm}$. The test disc diameter was $150 \mathrm{~mm}$, the thickness was $2 \mathrm{~mm}$, while 3 leaves were linear, the height was $16 \mathrm{~mm}$ over the center, and there was no eccentricity. The rice used for the tests was Huanghuazhan (water content: 15.2\%, sliding friction angle: $28.5^{\circ}$, weight of 1000 grains is $22.5 \mathrm{~g}$ ), which was filtered and cleaned. The sowing quantity of rice was $2 \mathrm{~kg}$, and the test time was $30 \mathrm{~s}$. The falling area of the disc was curved and the curvature of the falling area had a greater influence on the uniformity. We took the real-tested width and the curvature of the falling area as indicators. The width was measured as follows: based on the center line in front of the test bench where the disc was located, the inoculation cloth was tilted on the ground in front of the test bench, and the length of the seed falling belt perpendicular to the baseline was measured, indicating that the efficiency increases with the width. The curvature of the seed falling area was measured as follows: first, the end positions of the seed falling belt were determined and the two end points were marked, after which the maximum distance between the midpoint of the two end points and the arc of the seed falling belt were measured. This distance was the curvature of the belt. The shorter the distance, the smaller was the curvature of the drop.

4.1.1 Orthogonal test results and range analysis

The test and range analysis results are given in Tables 2 and 3, respectively. $K$ value is the sum of the results of various factors, $k$ is the average value of $K$ according to the optimal level of each factor that can be determined, and $R$ value is the difference between the maximum and minimum value of $k$, determining the primary and secondary order of the factors. The results showed that the main factors of the width were CBA, in order, the optimal combination was $\mathrm{C} 3 \mathrm{~B} 1 \mathrm{~A} 3$, the main factors impacting the curvature of seed falling area were $\mathrm{ACB}$, and, hence, the optimal combination was $\mathrm{A} 3 \mathrm{C} 3 \mathrm{~B} 3$. Moreover, Table 3 shows that the effects the levels of each factor had on the curvature of the seed falling area were small, so the ideal combination of the three factors was C3B1A3.

Table 2 Orthogonal test programs and results

\begin{tabular}{|c|c|c|c|c|c|}
\hline Test numbers & Radiuses of particles position (A)/mm & Offset angles(B)/( $\left.{ }^{\circ}\right)$ & Speeds $(\mathrm{C}) / \mathrm{r} \cdot \mathrm{min}^{-1}$ & Measured widths $/ \mathrm{m}$ & Chord lengths of particles falling area $/ \mathrm{m}$ \\
\hline 1 & $1(18)$ & $1(0)$ & $1(500)$ & 4.64 & 0.86 \\
\hline 2 & $1(18)$ & $2(30)$ & $2(750)$ & 4.78 & 0.89 \\
\hline 3 & $1(18)$ & $3(60)$ & $3(1000)$ & 4.81 & 1.01 \\
\hline 4 & $2(37)$ & $1(0)$ & $2(750)$ & 5.01 & 0.98 \\
\hline 5 & $2(37)$ & $2(30)$ & $3(1000)$ & 5.11 & 1.02 \\
\hline 7 & $3(55)$ & $1(0)$ & $3(1000)$ & 5.32 & 1.14 \\
\hline 8 & $3(55)$ & $2(30)$ & $1(500)$ & 4.46 & 1.10 \\
\hline 9 & $3(55)$ & $3(60)$ & $2(750)$ & 4.89 & 1.10 \\
\hline
\end{tabular}

Table 3 Results of range analysis

\begin{tabular}{|c|c|c|c|c|c|c|}
\hline & \multicolumn{3}{|c|}{ Measured width $/ \mathrm{m}$} & \multicolumn{3}{|c|}{ Chord length of particles falling area/m } \\
\hline & Factor A & Factor B & Factor C & Factor A & Factor B & Factor C \\
\hline K1 & 14.23 & 14.97 & 13.41 & 2.76 & 2.98 & 2.96 \\
\hline K2 & 14.43 & 14.35 & 14.68 & 3.00 & 3.01 & 2.97 \\
\hline $\mathrm{K} 3$ & 14.67 & 14.01 & 15.24 & 3.34 & 3.11 & 3.17 \\
\hline $\mathrm{k} 1$ & 4.74 & 4.99 & 4.47 & 0.92 & 0.99 & 0.99 \\
\hline $\mathrm{k} 2$ & 4.81 & 4.78 & 4.89 & 1.00 & 1.00 & 0.99 \\
\hline $\mathrm{k} 3$ & 4.89 & 4.67 & 5.08 & 1.11 & 1.04 & 1.06 \\
\hline Range $R$ & 0.15 & 0.32 & 0.61 & 0.19 & 0.05 & 0.07 \\
\hline Optimum level & A3 & B1 & $\mathrm{C} 3$ & A3 & B3 & $\mathrm{C} 3$ \\
\hline Order & & $\mathrm{CBA}$ & & & $\mathrm{ACB}$ & \\
\hline
\end{tabular}




\section{Experiment on distribution and sampling uniformity}

\subsection{Distribution area}

It can be seen from the table test that the falling area was not symmetrically distributed around the forward direction of the UH, but biased. As seen in Table 5, the effective width and effective offset distance at different locations were measured by tape. The effective width referred to the transverse propagation distance perpendicular to the forward direction, excluding the end area where the sowing quantity was less than the conventional rice seeding requirement $\left(180\right.$ grains $\left./ \mathrm{m}^{2}\right)$. The effective offset distance referred to the width relative to the effective sowing width on the left side of the forward direction.

Table 5 Distribution of seed falling regions

\begin{tabular}{|c|c|c|c|c|c|c|c|c|c|}
\hline \multirow{2}{*}{ Effective width/m } & \multicolumn{9}{|c|}{ Position of seed falling port } \\
\hline & 1 & 2 & 3 & 4 & 5 & 6 & 7 & 8 & 9 \\
\hline$G$ & 4.91 & 4.78 & 4.76 & 5.01 & 4.98 & 4.87 & 5.22 & 5.15 & 4.89 \\
\hline$G 1$ & 2.46 & 2.40 & 2.54 & 2.42 & 2.45 & 2.43 & 2.26 & 2.30 & 2.32 \\
\hline Offset distance & 0.005 & 0.01 & 0.16 & 0.085 & 0.04 & 0.005 & 0.35 & 0.275 & 0.125 \\
\hline
\end{tabular}

Note: $G$ is the effective width, $G 1$ is the offset distance of seed falling regions, along the left side of the UH, the offset distance is the absolute differences between the half effective width and effective offset distance; The disc speed is $750 \mathrm{r} / \mathrm{min}$.

Table 5 shows that, in the same direction, the offset width of the falling area increased by increasing the radius of the falling mouth and the offset angle of the falling export. The maximum offset distance was $0.35 \mathrm{~m}$ and the minimum value was $0.005 \mathrm{~m}$, and the effective offset distance was not drastically affected by the radius and the offset angle.

\subsection{Sowing uniformity test}

Based on the theoretical analysis of the factors affecting the sowing uniformity and the experimental study on the performance parameters of the sowing disc, the sowing device was mounted on the UH to test the sowing uniformity. Test material: $\mathrm{UH}$ (Xiangnong TXA-16 planting UH, the maximum load is $16 \mathrm{~kg}$, the ideal operating height is $1-3 \mathrm{~m}$, and the ideal operating speed is $6 \mathrm{~m} / \mathrm{s}$ ). The rotation speed of the sowing disc was $1000 \mathrm{r} / \mathrm{min}$, and the distance from the seed falling position to the center of the disc was $55 \mathrm{~cm}$ without bias. The rice (Huanghuazhan) was selected, soaked, and the sowing quantity was $1.33 \mathrm{~kg} / \mathrm{min}$. As shown in Figure 6, three rows of collection points (a total of 57) were initially set along the $\mathrm{UH}$ in the forward direction according to the range of the width obtained from the previous distribution tests and symmetrically distributed along the baseline of the UH trajectory. The distances between sampling points were $30 \mathrm{~cm} \times$ $50 \mathrm{~cm}$ (row $\times$ column). The test area and sampling points (size of $22.6 \mathrm{~cm} \times 16.4 \mathrm{~cm}$ ) are shown in Figure 7. On the test day, the wind level was 2 , the flight height of the UH was $1 \mathrm{~m}$, and the flight speed was $3 \mathrm{~m} / \mathrm{s}$.

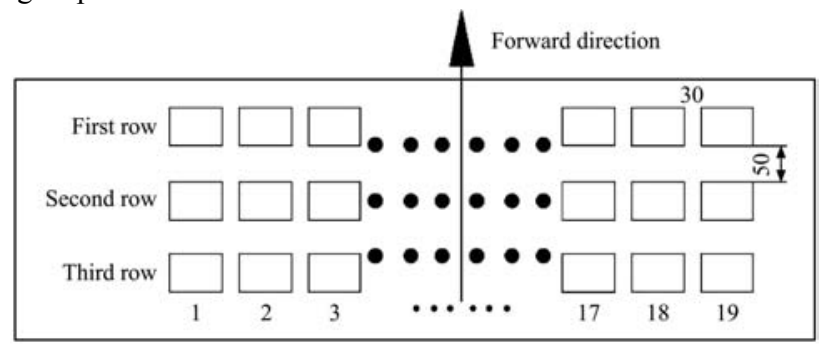

Figure 6 Positions of sample

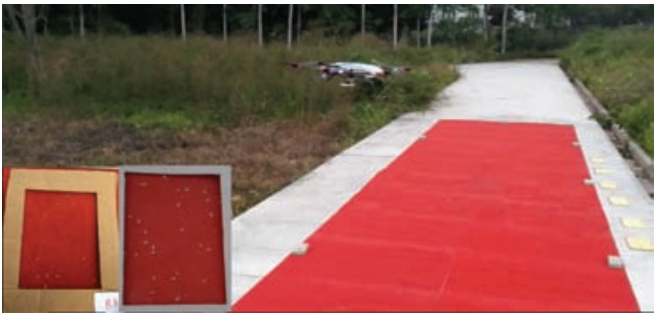

Figure 7 Real sampling points and test area
The number of falling grain particles was collected in different rows (Figures 8-10). Figure 8 shows that the particle distribution had a larger fluctuation in the first row (the maximum difference between the number of particles is 11). Figure 9 shows the particle distribution in the second row. On the left of the forward direction, the distribution curve was stable, contrary to the other side. Figure 10 shows the particle distribution in the third row. As a whole, the particle distribution had a smooth fluctuation, and the difference was not large, as shown in Figure 11. A statistical analysis of the number of particles at each collection point is shown in Table 6. As seen, the number of particles among the three rows was not very different, indicating good uniformity in the forward direction. The coefficient of variation of the number of particles in each row was more than $20 \%$, indicating that the uniformity in the horizontal direction was poor. Figure 7 shows the distribution of particles at each collection point. Specifically, the histogram represents the number of particles at each of the three collection points, and the curve represents the average of the number of particles in each column, excluding the two sampling points at both

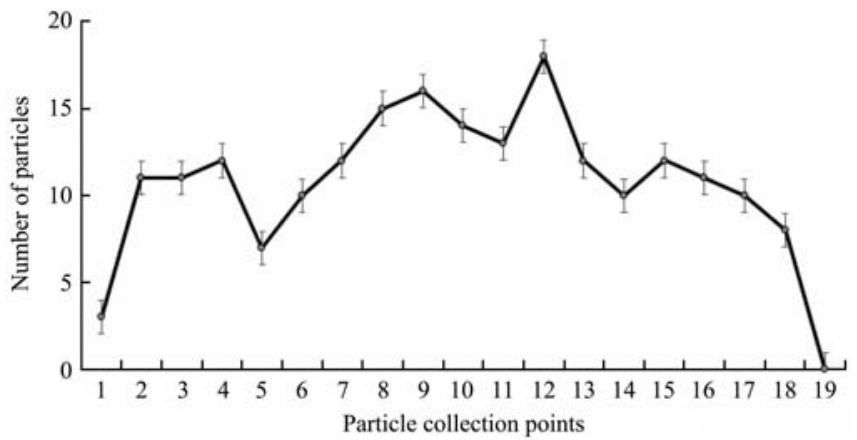

Figure 8 Statistics of particle counts in the first row

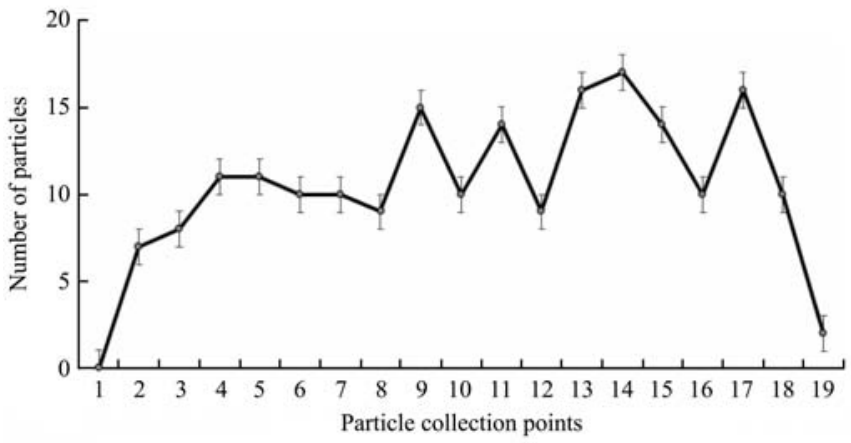

Figure 9 Statistics of particle counts in the second row 


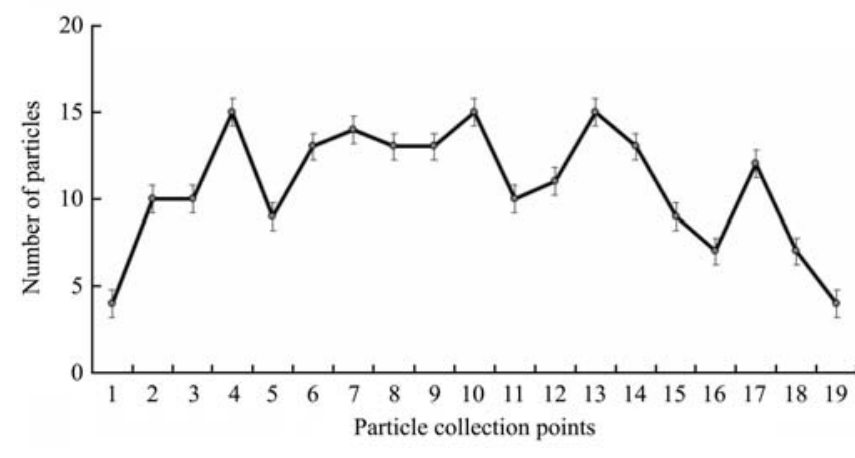

Figure 10 Statistics of particle counts in the third row

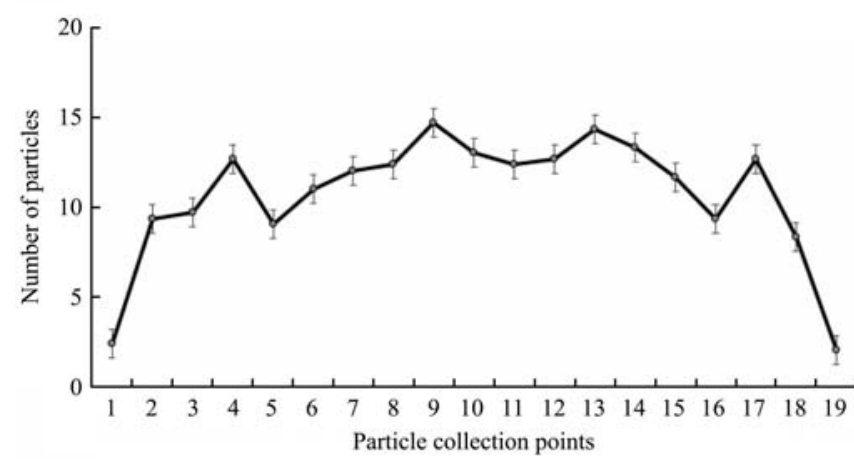

Figure 11 Average statistics of particle counts in all rows

Table 6 Uniformity analysis of particle counts in each collection point

\begin{tabular}{ccccc}
\hline Lines & average & difference & std. deviation & Coefficient of variation/\% \\
\hline 1 & 11.89 & 11 & 2.76 & 23.22 \\
2 & 11.59 & 10 & 3.08 & 26.62 \\
3 & 11.53 & 8 & 2.65 & 22.97 \\
\hline
\end{tabular}

ends. The reasons are as follows: 1) the air flow under the UH has a great influence on the seed particle movement; 2) the falling area of sowing is a complex superposition of the arc areas, which will surely cause differences in distribution of the number of particles in the grain falling area and affect the uniformity.

\section{Conclusions}

A centrifugal disc-type sowing device for an $\mathrm{UH}$ was designed. The rotation speed of the disc, the distance from the falling export to the center of the disc and the offset angle of the seed outlet were analyzed, and the optimal combination was established. Tests to determine the uniformity and the width of sowing were carried out.

The results showed the following conclusions:

1) The distance between the position of the falling mouth and the center of the disc (A), the offset angle of the exit position from the forward direction (B), and the rotation speed (C) had an effect on the sowing width in the descending order: $\mathrm{C}, \mathrm{B}, \mathrm{A}$, and had an effect on the curvature of the seed falling area was in the descending order: A, C, B. Between the two, the former was more important.

2) The rotational speed of the disc, the distance from the falling export to the center of the disk, and the offset angle of the falling mouth had little effect on the offset of the width.

3) The uniformity test on the ground showed that the coefficient of variation of uniformity in the transverse direction (perpendicular to the UH advancing direction) was more than $20 \%$, and the differences of the number of particles between the rows of the UH were not immense.
Though the centrifugal disc-type sowing device for $\mathrm{UH}$ functioned normally, the sowing scale was small, and the sowing uniformity should be improved. Moreover, the parameters of the key components such as the seeding device and the centrifugal disc need to be further optimized. The effects of the operation height during sowing operations need to be explored, and plenty of field test data to calibrate sowing parameters are also required, in order to consider more influencing factors impacting the field operations of the UH.

\section{Acknowledgments}

The authors acknowledge that this work was financially supported by the Science and Technology Plan of Guangdong Province of China (Project No. 2017B090907031, 2017B090903007, 2015B020206003, 2014B090904073), and Innovative Research Team of Guangdong Province Agriculture Research System (2017LM2153) for funding this research. The authors also thank the anonymous reviewers for their critical comments and suggestions to improve the manuscript.

\section{[References]}

[1] Luo X W. Thinking of speeding up the development of our country agricultural aviation technology. Agricultural Technology and Equipment, 2014; 5(281): 7-15. (in Chinese)

[2] Zhou Z Y, Zang Y, Luo X W, Lan Y B, Xue X Y. Technology innovation development strategy on agricultural aviation industry for plant protection in China. Transactions of the CSAE, 2013; 29(24): 1-10. (in Chinese)

[3] Zhang Y C. Sketch of the current development situation and technology innovation of the world agricultural aviation. Hunan Agricultural Mechinery, 2013; 4(2): 16-17. (in Chinese)

4] Zhang C. Development of the aircraft plant protection and agricultural aviation application technology in American. Journal of Era of Agricultural Machinery, 2015; 42(7): 168. (in Chinese)

[5] Zhang Y. The application of remote control helicopter agriculture in Japan. World Agriculture, 1997; 4: 49-50. (in Chinese)

[6] Zhang P, Zheng H G, Yu J X. Study on the improving technology of cold soak field in the high land area. Journal of Yunnan Agricultural University, 2005; 20(5): 665-670. (in Chinese)

[7] Jiao J G, Zhang H J, He D L. Character and improving measures of cold spring paddy soil in China. Journal of Agricultural Science, 2012; 40(7): 4247-4248. (in Chinese)

[8] Li C S, Tang Y L, Wu C, Huang G. Effect of sowing patterns on growth, development and yield formation of wheat in rice stubble land. Transactions of the CSAE, 2012; 28(18): 36-43. (in Chinese)

[9] Tang Y L, Li C S, Wu C, Wu X L, Huang G, Ma X L. Effects of sowing patterns on establishment quality, grain yield and production benefit of intercropping wheat in hilly countries. Chinese Agricultural Science, 2013; 46(24): 5089-5097. (in Chinese)

[10] Ren M E. Current Situation and Countermeasures of Mudflat Development and Utilization in China. Journal of Chinese Academy of Sciences, 1996; 14(6): 440-443. (in Chinese)

[11] Wan T B. Marsh resources and its development and protection in China Journal of World Environment, 1991; 1: 24-25. (in Chinese)

[12] Yang M L, Bai R P, Liu M, Tu Z Q. Development of agricultural mechanization and construxtion of modern agriculture. Transactions of the CSAM, 2005; 36(7): 68-72. (in Chinese)

[13] Chen D C, Liu W X. Discussion of the south rice mechanization production mode. Journal of Guangxi Agricultural Mechanization, 2000; 3: 4-6. (in Chinese)

[14] You G W. Discussion on the development suggestions and the problems existing in the mechanization of rice production. Agricultural Machinery Use and Maintenance, 2015; 12: 22-23. (in Chinese)

[15] Greipsson S, El-Mayas H. Large-scale reclamation of barren lands in iceland by aerial seeding. Land Degradation and Development, 1999; 10: 185-193.

[16] Xiao X, Wei X H, Liu Y Q, Ouyang X Z. Aerial seeding: An effective 
forest restoration method in highly degraded forest landscapes of sub-tropic regions. Forests, 2015; 6(6): 1748-1762.

[17] Xu F. Apply and market prospect of agricultural plant protection UAV. Agricultural Equipment Technology, 2016; 42(1): 49-51. (in Chinese)

[18] Lan Y B, Chen S D, Fritz B K. Current status and future trends of precision agricultural aviation technologies. Int J Agric \& Biol Eng, 2017; 10(3): $1-17$.

[19] Ruan X D. Agricultural UAV, the helper of modern agriculture. New Economy Weekly, 2015-04-05. (in Chinese)

[20] Zhao D W, Meng Y. Research on the mechanized precision seeding technology. Agricultural Science \& Technology and Equipment, 2010; 6: 58-60. (in Chinese)

[21] He X K, Bonds J, Herbst A, Langenakens J. Recent development of unmanned aerial vehicle for plant protection in East Asia. Int J Agric \& Biol Eng, 2017; 10(3): 18-30.

[22] Pan S Q. Experimental study of the horizontal disc type spreader parts. Changchun: Jilin Agricultural University, 2004. (in Chinese)

[23] Sun Q L, Zhao H L, Zhang X H. Current situation and development of seed sowing devices. Shandong Agricultural Mechinery, 2002; 2: 8-9. (in Chinese)
[24] Du X Q, Xiao M H, Hu X Q, Chen J N, Zhao Y. Numerical simulation and experiment of gas-solid two-phase flow in cross-flow grain cleaning device. Transactions of the CSAE, 2014; 30(3): 27-34. (in Chinese)

[25] Li J Y, Zhou Z Y, Hu L, Zang Y, Xu S, Liu A M. Optimization of operation parameters for supplementary pollination in hybrid rice breeding using round multi-axis multi-rotor electric unmanned helicopter. Transaction of the CSAE, 2014; 30(11): 1-9. (in Chinese)

[26] Zhao Y N, Sun Y Z, Mo D J. Research and development of a gas flow sensor with suspending gyrorotor. Journal of Beijing University of Chemical Technology, 1998; 25(4): 67-72. (in Chinese)

[27] Hu L, Zhou Z Y, Luo X W, Wang P, Yang Y A, Li J Y. Development and experiment of a wireless wind speed sensor network measurement system for unmanned helicopter. Transactions of the CSAM, 2014; 45(5): 221-226. (in Chinese)

[28] Deng C X, Tao D C, Gao J P. Dynamic characteristics and factors affecting performance of air-stream cleaning windmill. Transactions of the CSAE, 2006; 22(4): 121-125. (in Chinese)

[29] Ahmed B, Pota H R, Garratt M. Flight control of a rotary wing UAV using backstepping. International Journal of Robust \& Nonlinear Control, 2010; 20(6): 639-658. 